\title{
Comparison of the JNC7 and 2017 American College of Cardiology/American Heart Association Guidelines for the Management of Hypertension in Koreans: Analysis of Two Independent Nationwide Population-Based Studies
}

\author{
Won-Jun Choi $1,+\left(\mathbb{C}\right.$, Hye-Sun Lee ${ }^{2,+} \mathbb{C}^{\circ}$, Jung Hwa Hong ${ }^{3}$, Hyuk-Jae Chang ${ }^{4, *}$ a and \\ Ji-Won Lee ${ }^{5, *(\mathbb{D})}$ \\ 1 Family Medicine, Graduate School, Yonsei University College of Medicine, Seoul 03722, Korea; \\ slashwj@gmail.com \\ 2 Biostatistics Collaboration Unit, Yonsei University College of Medicine, Seoul 06273, Korea; \\ HSLEE1@yuhs.ac \\ 3 Institute of Health Insurance \& Clinical Research, National Health Insurance Service Ilsan Hospital, \\ Seoul 10444, Korea; jh_hong@nhimc.or.kr \\ 4 Division of Cardiology, Severance Cardiovascular Hospital, Yonsei University College of Medicine, Yonsei \\ University Health System, Seoul 03722, Korea \\ 5 Department of Family Medicine, Gangnam Severance Hospital, Yonsei University College of Medicine, \\ Seoul 06273, Korea \\ * Correspondence: hjchang@yuhs.ac (H.-J.C.); indi5645@yuhs.ac (J.-W.L.); Tel.: +82-2-2228-0919 (H.-J.C.); \\ +82-2-2019-2480 (J.-W.L.) \\ + These authors contributed equally to this work.
}

Received: 8 November 2019; Accepted: 14 December 2019; Published: 16 December 2019 updates

\begin{abstract}
The optimal blood pressure (BP) guidelines in Asian populations have not been determined. We compared all-cause and cardiovascular mortality based on the Joint National Committee 7 (JNC7) and 2017 American College of Cardiology/American Heart Association (ACC/AHA) guidelines. The National Health Insurance System-National Health Screening Cohort (NHIS-HEALS) and Korea National Health and Nutrition Examination Survey (KNHANES) were utilized. BPs were classified into three groups according to each guideline, and survival rates were analyzed with Kaplan-Meier curves and log-rank tests. Hazard ratios (HRs) were calculated using multivariable cox regression analyses, and the discriminatory ability for clinical outcomes was assessed by Harrell's C-indexes. The JNC7 guidelines demonstrated a linear association between BP levels and survival outcomes. Adjusted HRs from the JNC7 guidelines differentiated the hypertension group $(\geq 140 / 90)$ from the pre $(130 / 80-139 / 89)$ and normal $(<130$ and $<80)$ BP groups in clinical outcomes. In contrast, the 2017 ACC/AHA guidelines showed inconsistent survival outcomes according to BP classification (normal: $<120$ and $<80$, elevated: $120-129$, and $<80$, and HTN: $\geq 130 / 80$ ). According to Harrell's C-indexes, the JNC7 guidelines had greater discrimination ability in survival outcomes in the NHIS-HEALS dataset. Our results suggest that the JNC7 guidelines are more appropriate than the 2017 ACC/AHA guidelines in Korean populations.
\end{abstract}

Keywords: hypertension; mortality; guidelines; blood pressure

\section{Introduction}

In 2017, the American College of Cardiology/American Heart Association (ACC/AHA) published new blood pressure (BP) management guidelines that included changing the diagnostic standard 
for hypertension from a starting BP of $140 / 90 \mathrm{~mm} \mathrm{Hg}$, as in the Joint National Committee 7 (JNC7) guidelines, to a BP of 130/80 mm Hg. Additionally, the 2017 ACC/AHA guidelines recommend antihypertensive medication for adults at high risk of cardiovascular disease (CVD) with systolic BP (SBP) 130 to $139 \mathrm{~mm} \mathrm{Hg}$ or diastolic BP (DBP) 80 to $89 \mathrm{~mm} \mathrm{Hg}$ as well as treatment to lower SBP/DBP to $<130 / 80 \mathrm{~mm} \mathrm{Hg}$ for all adults taking antihypertensive medication [1].

The systolic blood pressure intervention trial (SPRINT) and recent meta-analysis revealed an intensive BP reduction with antihypertensive treatment was beneficial in reducing cardiovascular outcomes according to the new ACC/AHA guidelines [2,3]. Subsequent studies supported the appropriateness of the new guidelines and have shown a linear association between BP levels, all-cause mortality, and the risk of CVD with the lowest risk at a SBP level less than $130 \mathrm{~mm} \mathrm{Hg}$ [4,5].

In contrast, several studies, including the valsartan in elderly isolated systolic hypertension (VALISH) and action to control cardiovascular risk in diabetes (ACCORD) studies, failed to demonstrate the benefit of the new BP guidelines [6,7] and inconsistent findings make it difficult to broadly apply the 2017 ACC/AHA guidelines. Indeed, the 2018 European guidelines for the management of hypertension maintained the existing hypertension definition of $140 / 90 \mathrm{~mm} \mathrm{Hg}$ [8].

Guidelines for hypertension need to consider the disparity of country- or race-specific risk factors. $\mathrm{BP}$ related CVDs are more common in Asians than in Westerners [9]. However, it remains unknown whether intensive BP reduction leads to improved all-cause mortality and cardiovascular outcomes. Moreover, the optimal guidelines for discriminating high risk groups in terms of hypertension in Asian populations have not been determined.

Therefore, we aimed to compare all-cause and CVD mortality in a Korean population according to the JNC7 and 2017 ACC/AHA guidelines using two large nationally representative databases.

\section{Materials and Methods}

\subsection{Study Population and Data Collection}

\subsubsection{National Health Insurance System-National Health Screening Cohort (2006-2015 NHIS-HEALS)}

This study was based on data obtained from the NHIS-HEALS, a nationally retrospective cohort study conducted by the Korea Centers for Disease Control and Prevention. The NHIS is a universal health coverage program, and all insured individuals and their dependents are required to undergo general health examinations every 2 years. The National Health Examination followed standardized procedures, and its validity is described elsewhere [10].

Study populations were followed from 1 January 2006 until the date of a cardiovascular event, death, or 31 December 2015, whichever came first. We extracted 1,021,208 participants aged 30-74 years whose data were available and excluded individuals who met any of the following criteria $(n=878,590)$ : Younger than 30 or older than 75 years of a age; history of hospitalization for a diagnosis of myocardial infarction (MI; Korean Standard Classification of Diseases, KCD codes I21-I23) or stroke (KCD codes I60-I64); any type of malignant cancer; death in the year of enrolment; single medical record after 2006; and those with missing SBP, DBP, or death data. Following these exclusions 142,618 participants were included in the final analysis as shown in Figure 1. 
A.

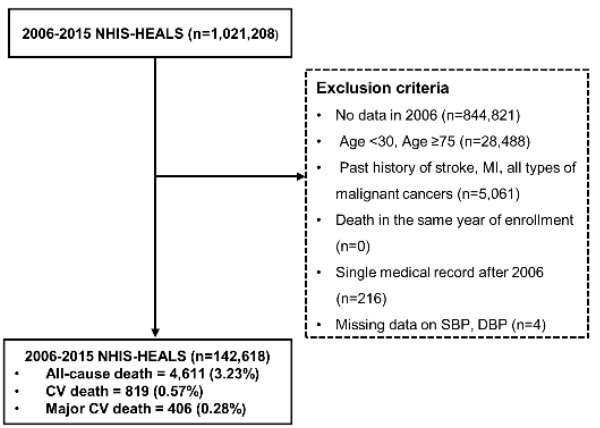

B.

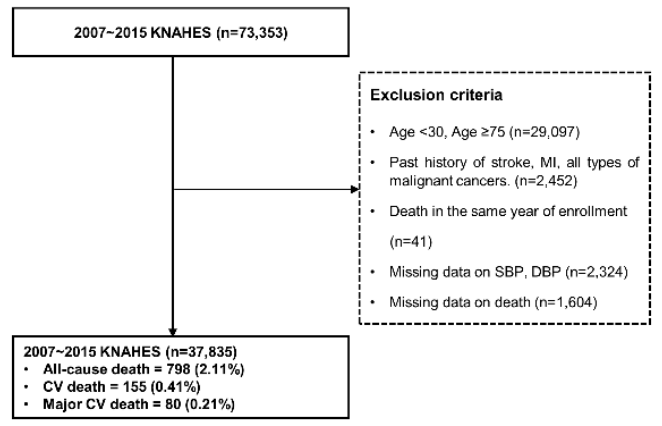

Figure 1. Flow chart of the study population. A: 2006-2015 NHIS-HEALS; B: 2007-2015 KNAHES.

Self-reported cigarette smoking and alcohol consumption were determined by questionnaire. Each participant was categorized as a non-smoker, ex-smoker, or current smoker with respect to smoking status. Participants were categorized as non-drinkers, intermittent drinkers $(\leq 3-4$ times a week), or daily drinkers with respect to alcohol use. Physical activity was divided into five groups according to the amount of exercise per week, and household income was divided into five groups based on the 10th quantile information.

BP was measured at local hospitals, each of which met the internal and external quality control procedures of the Korean Association of External Quality Assessment Service. After participants rested while seated for at least $2 \mathrm{~min}$, and BP measurements were taken by digital or automatic monitors during the health examination. All BP measurements, including BP data before the index period, were used to calculate mean BP.

\subsubsection{Korea National Health and Nutrition Examination Survey (2007-2015 KNHANES Cohort)}

We obtained data from the 2007-2015 Korean National Health and Nutrition Examination Survey (KNHANES) cohort, a nationally representative survey conducted by the Korean Ministry of Health and Welfare. KNHANES datasets have been provided publicly, 10 but recent datasets have been further matched with death information from any cause. As a result, the data can be used for nationally representative death statistics. Study populations were followed from 1 January 2007 to 31 December 2015.

We extracted 73,353 participants aged 30-74 years and excluded participants who met any of the following criteria ( $n=35,518$ ): Participants younger than 30 or older than 75 years, a history of stroke, acute MI, or any type of malignant cancer, death in the year of enrollment, and those with missing SBP, DBP, or death data. Following these exclusions 37,835 participants were included in the final analysis as shown in Figure 1.

Self-reported cigarette smoking and alcohol consumption were determined by questionnaire. Each participant was categorized as a non-smoker, ex-smoker, or current smoker with respect to smoking status. Participants were also asked about the frequency of their alcohol intake and weekly physical activity. Alcohol use was defined if one of two criteria was satisfied: Drinking quantity ( $\geq 7$ drinks for males and $\geq 5$ drinks for females) and frequency ( $\geq 2$ times per week).

BP measurements were taken at local examination centers by trained examiners. BP was measured three times, and the average of the second and third measurements was used for analysis [10].

\subsection{Outcome Measurement}

The primary outcomes of the study were all-cause mortality and all cardiovascular mortality. All cardiovascular mortality was defined as death from a disease of the circulatory system (KCD codes I00-I99). We selected myocardial infarction (MI, KCD codes I21-I23), hemorrhagic stroke 
(KCD codes I60-I62), and ischemic stroke (KCD code I63) for inclusion among the detailed causes of cardiovascular mortality.

\subsection{Statistical Analysis}

BPs were classified into three groups according to each guideline. Under the JNC7 guidelines, BPs were categorized as follows: normal $(<130$ and $<80)$, pre-hypertension (130/80-139/89), and hypertension (HTN, $\geq 140 / 90)$. Under the 2017 ACC/AHA guidelines, BPs were classified as normal $(<120$ and $<80)$, elevated $(120-129$ and $<80)$, and HTN $(\geq 130 / 80)$. The characteristics of the study population were presented as means \pm standard deviations and frequencies (percentages). Groups were compared using one-way analysis of variance (ANOVA) for continuous variables and chi-square tests for categorical variables.

The survival rates of each group according to the adjustment criteria presented in each guideline were analyzed by Kaplan-Meier curves and log-rank tests. The warranty period was defined as the time required for the cumulative mortality incidence to reach $0.5 \%$ for each group. If the $0.5 \%$ threshold was not met, the value was expressed as the time of the last follow-up. In addition, incidence per 1000 person-years was calculated for each group. The hazard ratios (HRs), $95 \%$ confidence intervals (CIs), and $p$ values for trends with reference to the pre-HTN group (130/80-139/89) in the JNC7 guidelines and the elevated BP group (120-129 and <80) in the 2017 ACC/AHA guidelines were calculated using multivariable Cox regression analyses after adjusting for age, sex, and body mass index (BMI) in the KNHANES cohort and age, sex, BMI, physical activity, household income, smoking status, alcohol status, fasting serum glucose, and total cholesterol in the NHIS-HEALS cohort. The KNAHES cohort had a smaller number of events compared to the NHIS-HEALS cohort. Therefore, we adjusted the KNAHES cohort for the smallest exploratory variable, while the NHIS-HEALS cohort was adjusted for additional variables. To evaluate the predictability of the JNC7 and 2017 ACC/AHA guidelines, we calculated Harrell's c-index (95\% CI). To calculate the 95\% CIs and $p$-values for Harrell's C-index and the differences between JNC7 and ACC/AHA, we used a bootstrap method and resampled 1000 times.

The study was approved by the Institutional Review Board of Yonsei University Health System (IRB number: 3-2018-0160), and the requirement for informed consent was waived. All statistical analyses were performed using SAS software, version 9.4 (SAS Institute Inc., Cary, NC, USA). All statistical tests were two-sided, and statistical significance was determined at $p<0.05$.

\section{Results}

Using the NHIS-HEALS and KNAHES datasets of 1,021,208 and 73,353 individuals, respectively, we identified 142,618 and 37,835 adults for inclusion in this study. The number of all-cause mortality (ACM), all cardiovascular deaths (ACD), and major cardiovascular deaths (MACE; acute MI, ischemic stroke, and hemorrhagic stroke) in each dataset are shown in Figure 1. During follow-up, 4611 ACM and 819 CVD events occurred in the NHIS-HEALS cohort, and 789 ACM and 155 CVD events occurred in the KNAHES cohort. Table 1 presents the baseline characteristics according to BP levels under the two different criteria. The prevalence of hypertension was estimated to be $40.64 \%$ (NHIS-HEALS) and $44.8 \%$ (KNAHES) based on the 2017 ACC/AHA guidelines, which was a dramatic increase compared to the $9.64 \%$ (NHIS-HEALS) and $18.4 \%$ (KNAHES) prevalence rates based on the JNC7 guidelines. Individuals with a higher BP tended to be older, consumed more alcohol, had a higher BMI, and had higher fasting serum glucose levels in both cohorts.

Figure 2 shows the cumulative incidence of the three outcomes (ACM, ACD, and MACE) for each guideline using Kaplan-Meier curves and log-rank tests. Based on the JNC7 guidelines, there was a significant linear trend towards an increased risk of all three outcomes with worse BP control regardless of dataset. The 2017 ACC/AHA guidelines, however, showed different results. In the NHIS-HEALS dataset, the 2017 ACC/AHA guidelines showed increased incidence of all three outcomes in sequential BP groups, but in the KNAHES dataset, the cumulative incidence of ACM, ACD, and MACE was highest in the elevated BP group, followed by the HTN and normal BP groups. 
Table 1. Baseline characteristics according to JNC7 and 2017 ACC/AHA guidelines.

\begin{tabular}{|c|c|c|c|c|c|c|c|c|}
\hline \multirow{2}{*}{$\begin{array}{c}\text { Guidelines } \\
\text { 2006-2015 NHIS-HEALS }\end{array}$} & \multicolumn{3}{|c|}{ JNC7 Guidelines } & \multicolumn{5}{|c|}{2017 ACC/AHA Guidelines } \\
\hline & $\begin{array}{c}<130 \text { and }<80 \\
(n=84,653)\end{array}$ & $\begin{array}{c}130 / 80-139 / 89 \\
(n=44,214)\end{array}$ & $\begin{array}{c}\geq 140 / 90 \\
(n=13,751)\end{array}$ & $p$-Value ${ }^{1}$ & $\begin{array}{c}<120 \text { and }<80 \\
(n=53,374)\end{array}$ & $\begin{array}{c}120-129 \text { and } \\
<80(n=31,279)\end{array}$ & $\begin{array}{c}\geq 130 / 80 \\
(n=57,965)\end{array}$ & $p$-Value ${ }^{1}$ \\
\hline Age & $45.53 \pm 10.52$ & $49.36 \pm 11.26$ & $53.92 \pm 11.94$ & $<0.0001$ & $44.04 \pm 9.7$ & $48.09 \pm 11.35$ & $50.45 \pm 11.58$ & $<0.0001$ \\
\hline Female Sex, N (\%) & $38948(46.01)$ & $14,321(32.39)$ & $5023(36.53)$ & $<0.0001$ & $27,377(51.29)$ & $11571(36.99)$ & $19344(33.37)$ & $<0.0001$ \\
\hline Height, $\mathrm{m}^{2}$ & $163.76 \pm 8.81$ & $164.63 \pm 9.15$ & $162.85 \pm 9.38$ & $<0.0001$ & $163.42 \pm 8.59$ & $164.35 \pm 9.15$ & $164.21 \pm 9.24$ & $<0.0001$ \\
\hline Weight, kg & $62.46 \pm 10.46$ & $67.11 \pm 11.21$ & $67.14 \pm 11.94$ & $<0.0001$ & $60.87 \pm 10.04$ & $65.17 \pm 10.59$ & $67.11 \pm 11.39$ & $<0.0001$ \\
\hline BMI, $\mathrm{kg} / \mathrm{m}^{2}$ & $23.21 \pm 2.84$ & $24.67 \pm 2.98$ & $25.21 \pm 3.24$ & $<0.0001$ & $22.71 \pm 2.74$ & $24.05 \pm 2.81$ & $24.8 \pm 3.05$ & $<0.0001$ \\
\hline Physical activity, N (\%) & & & & $<0.0001$ & & & & $<0.0001$ \\
\hline 0 & $43,192(52.57)$ & $20,980(48.83)$ & $6884(51.54)$ & & $28,066(54.2)$ & $15126(49.79)$ & $27,864(49.47)$ & \\
\hline $1-2$ & $23,590(28.71)$ & $13,070(30.42)$ & $3533(26.45)$ & & $14,606(28.21)$ & $8984(29.57)$ & $16,603(29.48)$ & \\
\hline $3-4$ & $9530(11.6)$ & $5235(12.18)$ & $1504(11.26)$ & & $5856(11.31)$ & 3674 (12.09) & $6739(11.97)$ & \\
\hline $5-6$ & $2141(2.61)$ & $1150(2.68)$ & $380(2.85)$ & & $1254(2.42)$ & $887(2.92)$ & $1530(2.72)$ & \\
\hline Almost everyday & $3708(4.51)$ & $2531(5.89)$ & $1055(7.9)$ & & $2001(3.86)$ & $1707(5.62)$ & $3586(6.37)$ & \\
\hline Household income, $\mathrm{N}(\%)$ & & & & $<0.0001$ & & & & $<0.0001$ \\
\hline $1-2$ & $11,982(14.41)$ & $6366(14.66)$ & $2279(16.74)$ & & $7450(14.18)$ & $4532(14.79)$ & 8645 (15.16) & \\
\hline $3-4$ & $11,231(13.5)$ & $5834(13.44)$ & $2056(15.1)$ & & $7084(13.49)$ & $4147(13.53)$ & $7890(13.84)$ & \\
\hline $5-6$ & $13,664(16.43)$ & 7278 (16.77) & $2438(17.91)$ & & $8407(16.01)$ & $5257(17.16)$ & $9716(17.04)$ & \\
\hline $7-8$ & $22,734(27.34)$ & $11,374(26.2)$ & $3340(24.53)$ & & $14,441(27.49)$ & $8293(27.07)$ & $14,714(25.8)$ & \\
\hline $9-10$ & $23,555(28.32)$ & $12,559(28.93)$ & $3503(25.73)$ & & $15,143(28.83)$ & $8412(27.45)$ & $16,062(28.17)$ & \\
\hline Smokers, N (\%) & $27,345(33.27)$ & $17,041(39.69)$ & $4827(36.24)$ & $<0.0001$ & $15,827(30.55)$ & $11518(37.92)$ & $21868(38.87)$ & $<0.0001$ \\
\hline \multicolumn{9}{|l|}{ Alcohol drinkers, N (\%) } \\
\hline Non-drinker & $45,514(54.86)$ & $20,411(47.07)$ & $6732(50.1)$ & & $29,806(57.03)$ & 15708 (51.17) & $27,143(47.79)$ & \\
\hline Intermittent drinker & $35,851(43.22)$ & $21,417(49.39)$ & $6007(44.71)$ & & $21,708(41.54)$ & $14143(46.07)$ & $27,424(48.28)$ & \\
\hline Daily drinker & $1593(1.92)$ & $1535(3.54)$ & $697(5.19)$ & & $747(1.43)$ & $846(2.76)$ & $2232(3.93)$ & \\
\hline $\mathrm{SBP}, \mathrm{mm} \mathrm{Hg}$ & $116.16 \pm 11.73$ & $130.75 \pm 12.3$ & $146.66 \pm 16.25$ & $<0.0001$ & $111.83 \pm 10.23$ & $123.55 \pm 10.36$ & $134.52 \pm 14.96$ & $<0.0001$ \\
\hline $\mathrm{DBP}, \mathrm{mm} \mathrm{Hg}$ & $72.67 \pm 8.2$ & $82.72 \pm 8.34$ & $90.37 \pm 11.06$ & $<0.0001$ & $70.64 \pm 7.87$ & $76.13 \pm 7.55$ & $84.53 \pm 9.63$ & $<0.0001$ \\
\hline Fasting Glucose, mg/dL & $93.47 \pm 22.22$ & $98.08 \pm 25.49$ & $103.91 \pm 32.39$ & $<0.0001$ & $91.79 \pm 20.37$ & $96.33 \pm 24.8$ & $99.46 \pm 27.4$ & $<0.0001$ \\
\hline Total cholesterol, mg/dL & $192.03 \pm 35.65$ & $200.21 \pm 36.33$ & $204.12 \pm 38.42$ & $<0.0001$ & $189.15 \pm 34.95$ & $196.95 \pm 36.3$ & $201.14 \pm 36.87$ & $<0.0001$ \\
\hline $\mathrm{AST}, \mathrm{mg} / \mathrm{dL}$ & $24.6 \pm 17.94$ & $27.32 \pm 23.45$ & $29.21 \pm 22.55$ & $<0.0001$ & $23.7 \pm 14.59$ & $26.14 \pm 22.45$ & $27.77 \pm 23.26$ & $<0.0001$ \\
\hline $\mathrm{ALT}, \mathrm{mg} / \mathrm{dL}$ & $24.12 \pm 22.32$ & $29 \pm 28.98$ & $30.15 \pm 26.87$ & $<0.0001$ & $22.58 \pm 20.05$ & $26.75 \pm 25.53$ & $29.27 \pm 28.5$ & $<0.0001$ \\
\hline
\end{tabular}


Table 1. Cont.

\begin{tabular}{|c|c|c|c|c|c|c|c|c|}
\hline \multirow{2}{*}{$\begin{array}{c}\text { Guidelines } \\
\begin{array}{c}\text { 2007-2015 KNAHES } \\
\text { Cohort }\end{array}\end{array}$} & \multicolumn{3}{|c|}{ JNC7 Guidelines } & \multicolumn{5}{|c|}{2017 ACC/AHA Guidelines } \\
\hline & $\begin{array}{c}<130 \text { and }<80 \\
(n=20,903)\end{array}$ & $\begin{array}{c}130 / 80-139 / 89 \\
(n=9983)\end{array}$ & $\begin{array}{c}\geq 140 / 90 \\
(n=6949)\end{array}$ & $p$-value ${ }^{1}$ & $\begin{array}{c}<120 \text { and }<80 \\
(n=17,989)\end{array}$ & $\begin{array}{c}120-129 \text { and } \\
<80(n=2914)\end{array}$ & $\begin{array}{c}\geq 130 / 80 \\
(n=16,932)\end{array}$ & $p$-value ${ }^{1}$ \\
\hline Age & $48.4 \pm 12.5$ & $52.0 \pm 11.9$ & $55.4 \pm 11.6$ & $<0.0001$ & $46.765 \pm 11.9$ & $58.469 \pm 11.709$ & $53.439 \pm 11.933$ & $<0.0001$ \\
\hline Female Sex, N (\%) & $13704(65.56)$ & $4714(47.22)$ & 3175 (45.69) & $<0.0001$ & $11,986(66.63)$ & $1718(58.96)$ & $7889(46.59)$ & $<0.0001$ \\
\hline BMI, $\mathrm{kg} / \mathrm{m}^{2}$ & $23.2 \pm 3.1$ & $24.5 \pm 3.3$ & $25.1 \pm 3.3$ & $<0.0001$ & $5623(32.20)$ & $1039(36.96)$ & $7504(45.87)$ & $<0.0001$ \\
\hline Physical activity, N (\%) & $1233(6.15)$ & $603(6.31)$ & $430(6.46)$ & 00.6341 & $2266(6.25)$ & $1080(6.25)$ & $153(5.52)$ & 0.2307 \\
\hline Household income, N (\%) & & & & $<0.0001$ & & & & $<0.0001$ \\
\hline Q1 & $2988(14.49)$ & $1654(16.83)$ & $1616(23.63)$ & & $2199(12.39)$ & $789(27.51)$ & $3270(19.62)$ & \\
\hline$\widehat{\mathrm{Q}} 2$ & $5277(25.60)$ & $2518(25.63)$ & $1829(26.74)$ & & $4464(25.15)$ & $813(28.35)$ & $4347(26.08)$ & \\
\hline Q3 & $6128(29.72)$ & $2735(27.83)$ & $1764(25.79)$ & & $5415(30.51)$ & $713(24.86)$ & $4499(27.00)$ & \\
\hline$\tilde{\mathrm{Q}} 4$ & $6224(30.19)$ & $2919(29.71)$ & $1630(23.83)$ & & $5671(31.95)$ & $553(19.28)$ & $4549(27.30)$ & \\
\hline Smokers, N (\%) & $6662(32.86)$ & $4361(45.19)$ & $3143(46.85)$ & $<0.0001$ & $7573(45.48)$ & $978(37.94)$ & $7085(48.50)$ & $<0.0001$ \\
\hline Alcohol drinkers, N (\%) & $8551(44.46)$ & $4234(48.50)$ & $2851(48.51)$ & $<0.0001$ & $23.072 \pm 3.072$ & $24.238 \pm 3.151$ & $24.751 \pm 3.295$ & $<0.0001$ \\
\hline $\mathrm{SBP}, \mathrm{mm} \mathrm{Hg}$ & $108.22 \pm 9.639$ & $124.22 \pm 8.702$ & $143.71 \pm 14.043$ & $<0.0001$ & $105.71 \pm 7.833$ & $123.71 \pm 2.93$ & $132.22 \pm 14.75$ & $<0.0001$ \\
\hline $\mathrm{DBP}, \mathrm{mm} \mathrm{Hg}$ & $69.876 \pm 6.256$ & $81.892 \pm 4.81$ & $90.245 \pm 9.73$ & $<0.0001$ & $69.367 \pm 6.233$ & $73.017 \pm 5.429$ & $85.32 \pm 8.329$ & $<0.0001$ \\
\hline Pulse rate & $57.557 \pm 12.041$ & $57.992 \pm 12.185$ & $60.412 \pm 16.426$ & 0.0002 & $57.531 \pm 11.812$ & $57.659 \pm 12.921$ & $59.042 \pm 14.225$ & 0.0217 \\
\hline Fasting Glucose, $\mathrm{mg} / \mathrm{dL}$ & $96.55 \pm 21.92$ & $101.61 \pm 24.799$ & $104.42 \pm 25.985$ & $<0.0001$ & $95.269 \pm 20.484$ & $104.52 \pm 28.035$ & $102.76 \pm 25.328$ & $<0.0001$ \\
\hline $\mathrm{HbA} 1 \mathrm{c}, \mathrm{mg} / \mathrm{dL}$ & $5.827 \pm 0.946$ & $5.981 \pm 1.005$ & $6.12 \pm 1.081$ & $<0.0001$ & $5.765 \pm 0.897$ & $6.17 \pm 1.124$ & $6.036 \pm 1.038$ & $<0.0001$ \\
\hline Insulin, $\mathrm{mg} / \mathrm{dL}$ & $9.209 \pm 6.171$ & $10.146 \pm 6.963$ & $10.575 \pm 7.307$ & $<0.0001$ & $9.03 \pm 5.71$ & $10.361 \pm 8.48$ & $10.328 \pm 7.114$ & $<0.0001$ \\
\hline Total cholesterol, mg/dL & $187.28 \pm 34.381$ & $195.58 \pm 35.902$ & $199.46 \pm 38.069$ & $<0.0001$ & $186.61 \pm 34.123$ & $191.4 \pm 35.675$ & $197.17 \pm 36.854$ & $<0.0001$ \\
\hline Triglycerides, mg/dL & $120.61 \pm 88.481$ & $154.95 \pm 124.52$ & $174.44 \pm 142.33$ & $<0.0001$ & $116.98 \pm 86.061$ & $143.13 \pm 99.322$ & $162.93 \pm 132.45$ & $<0.0001$ \\
\hline HDL-cholesterol, mg/dL & $50.173 \pm 11.786$ & $48.489 \pm 11.6$ & $48.216 \pm 11.583$ & $<0.0001$ & $50.481 \pm 11.828$ & $48.248 \pm 11.331$ & $48.377 \pm 11.594$ & $<0.0001$ \\
\hline LDL-cholesterol, mg/dL & $113.09 \pm 31.369$ & $116.23 \pm 34.847$ & $116.34 \pm 38.254$ & $<0.0001$ & $112.82 \pm 30.927$ & $114.73 \pm 33.952$ & $116.28 \pm 36.283$ & $<0.0001$ \\
\hline $\mathrm{AST}, \mathrm{mg} / \mathrm{dL}$ & $21.302 \pm 11.932$ & $24.121 \pm 14.125$ & $25.489 \pm 14.695$ & $<0.0001$ & $20.962 \pm 11.371$ & $23.416 \pm 14.781$ & $24.681 \pm 14.376$ & $<0.0001$ \\
\hline $\mathrm{ALT}, \mathrm{mg} / \mathrm{dL}$ & $19.931 \pm 18.982$ & $24.604 \pm 18.695$ & $25.672 \pm 17.601$ & $<0.0001$ & $19.613 \pm 18.845$ & $21.904 \pm 19.698$ & $25.042 \pm 18.262$ & $<0.0001$ \\
\hline BUN, mg/dL & $14.144 \pm 4.162$ & $14.783 \pm 4.362$ & $15.098 \pm 4.576$ & $<0.0001$ & $13.953 \pm 4.065$ & $15.326 \pm 4.545$ & $14.912 \pm 4.454$ & $<0.0001$ \\
\hline Creatinine, $\mathrm{mg} / \mathrm{dL}$ & $0.812 \pm 0.228$ & $0.865 \pm 0.259$ & $0.869 \pm 0.283$ & $<0.0001$ & $0.808 \pm 0.219$ & $0.837 \pm 0.276$ & $0.867 \pm 0.269$ & $<0.0001$ \\
\hline $\mathrm{WBC}, \mathrm{mg} / \mathrm{dL}$ & $5.951 \pm 1.693$ & $6.263 \pm 1.761$ & $6.423 \pm 1.781$ & $<0.0001$ & $5.915 \pm 1.685$ & $6.175 \pm 1.73$ & $6.328 \pm 1.771$ & $<0.0001$ \\
\hline
\end{tabular}

Data are expressed as the mean \pm SD or frequency (percentage). ${ }^{1}$ p-values were calculated using one-way ANOVA, Chi-square test, and post-hoc analysis. Abbreviations: JNC7, the Joint National Committee 7; 2017 ACC/AHA, 2017 American College of Cardiology/American Heart Association; SBP, Systolic blood pressure; DBP, diastolic blood pressure; AST, Aspartate transaminase; ALT, Alanine aminotransferase; BUN, Blood Urea Nitrogen; WBC, White blood cell. 

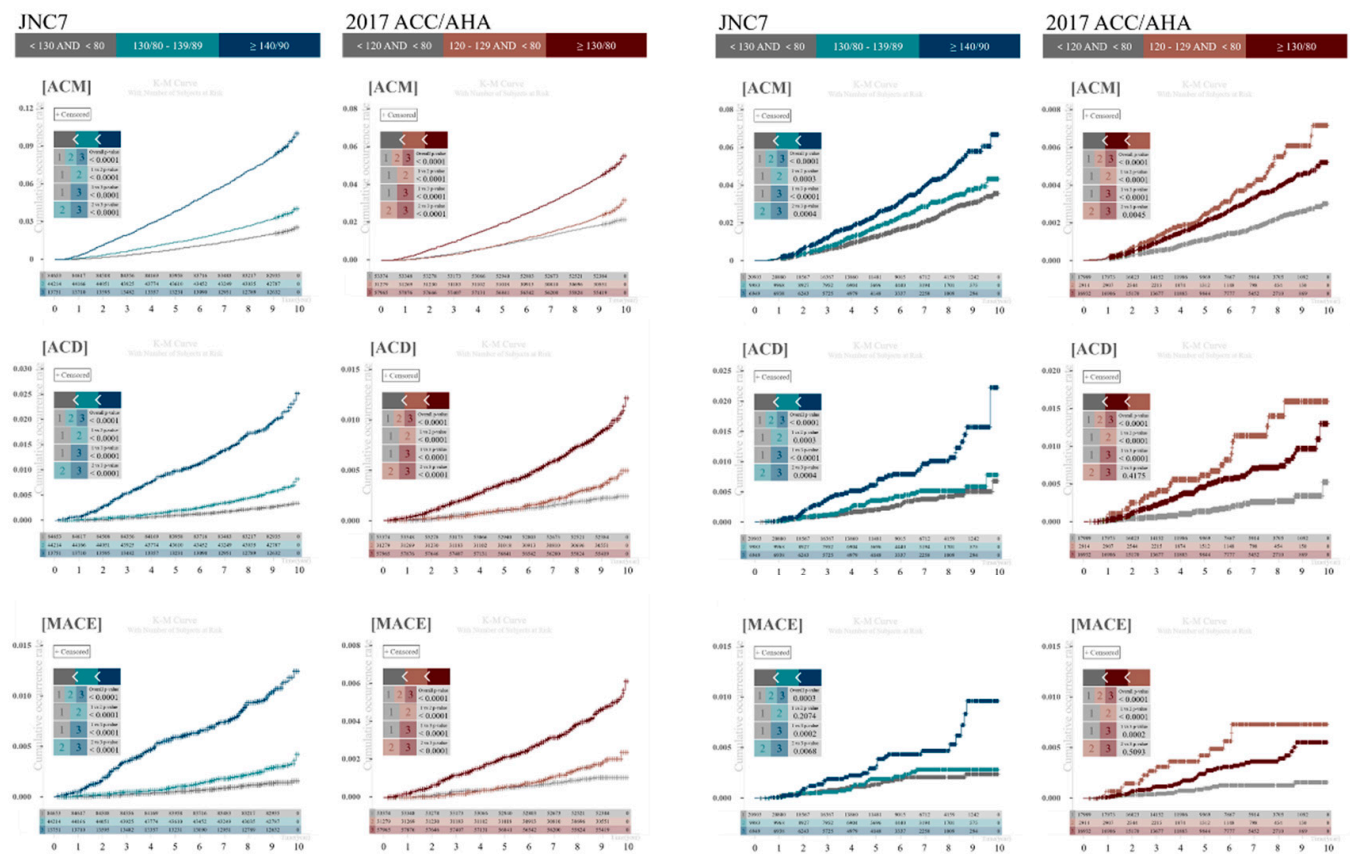

Figure 2. Kaplan-Meier curves with log-rank tests for the cumulative incidence of for all-cause mortality, all cardiovascular death, and major cardiovascular death according to JNC7 and 2017 ACC/AHA guidelines.

The warranty periods were defined as the duration in years that the cumulative mortality rate remained $<0.5 \%$. Based on the JNC7 guidelines, the HTN group had an unfavorable warranty period in all three outcomes compared to the elevated BP and normal BP groups in the two datasets. However, according to the 2017 ACC/AHA guidelines, results were inconsistent between the NHIS-HEALS and KNAHES datasets. Although the warranty periods for ACM and ACD were shortest in the HTN group in the NHIS-HEALS dataset, the elevated BP group had the shortest warranty period for ACM and ACD in the KNAHES dataset. Based on the JNC7 guidelines, the event rates for ACM, ACD, and MACE per 1000 person-years increased linearly with increasing BP in both datasets. However, according to the 2017 ACC/AHA guidelines, the cumulative incidence of ACM, ACD, and MACE was highest in the elevated BP group, followed by the HTN and normal BP groups in the KNAHES dataset. In other words, the 2017 ACC/AHA guidelines did not show linearity in warranty periods and event rates per 1000 person-years Table 2.

Table 3 shows adjusted HRs for ACM, ACD, and MACE according to each guideline after adjusting for all variables. Based on the JNC7 guidelines, the hazard ratio (95\% CIs) was statistically significantly higher in the HTN group in all three outcomes compared to the other two groups in both datasets: ACM 1.84 (1.67-2.00), ACD 2.72 (2.26-3.27), and MACE 3.25 (2.51-4.21) in the NHIS-HEALS dataset and ACM 1.24 (1.05-1.48), ACD 1.83 (1.26-2.65), and MACE 1.73 (1.04-2.90) in the KNAHES dataset. Unlike the JNC7 guidelines, the 2017 ACC/AHA guidelines showed different results between the two datasets. The HTN group showed the highest hazard ratio in all three outcomes in the NHIS-HEALS dataset: ACM 1.15 (1.06-1.24), ACD 1.76 (1.42-2.17), and MACE 2.09 (1.53-2.84). However, there were no statistically significant differences between the normal group and the elevated BP group for ACD or MACE in the NHIS-HEALS dataset. Conversely, in the KNAHES dataset, the hazard ratio for ACM was significantly higher in the normal group than in the elevated BP group according to the 2017 ACC/AHA guidelines. Moreover, based on the 2017 ACC/AHA guidelines, there was no statistically significant difference for all three HRs between the elevated BP group and the HTN group in the KNAHES dataset. Instead, the normal group had the lowest HRs for all three outcomes in the KNAHES dataset. 
Table 2. Warranty periods for all-cause mortality, all cardiovascular death, and major cardiovascular death according to JNC7 and 2017 ACC/AHA guidelines.

\begin{tabular}{|c|c|c|c|c|c|c|c|c|c|c|c|c|}
\hline \multicolumn{3}{|c|}{ Guidelines } & \multicolumn{5}{|c|}{ 2006-2015 NHIS-HEALS } & \multicolumn{5}{|c|}{ 2007-2015 KNAHES Cohorts } \\
\hline & & Groups & $\begin{array}{c}\text { Warranty } \\
\text { Period }(0.5 \%)\end{array}$ & $n$ & $\begin{array}{l}\text { Person-Time } \\
\quad \text { (Years) }\end{array}$ & $\begin{array}{c}\text { Events, } \mathrm{N} \\
(\%)\end{array}$ & $\begin{array}{c}\text { Incidence per } \\
1000 \text { Person-Years } \\
(95 \% \text { CI })\end{array}$ & $\begin{array}{c}\text { Warranty } \\
\text { Period }(0.5 \%)\end{array}$ & $n$ & $\begin{array}{l}\text { Person-Time } \\
\quad \text { (Years) }\end{array}$ & $\begin{array}{c}\text { Events, } \mathrm{N} \\
(\%)\end{array}$ & $\begin{array}{c}\text { Incidence per } \\
1000 \text { Person-Years } \\
(95 \% \text { CI) }\end{array}$ \\
\hline \multirow{9}{*}{ JNC7 } & $\mathrm{ACM}$ & $<130$ and $<80$ & 3.59 & 84,653 & $784,456.07$ & 1854 (2.19) & $2.36(0.15-4.57)$ & 2.45 & 20,903 & $11,2374.2$ & $352(1.68)$ & $3.13(2.81-3.46)$ \\
\hline & & $130 / 80-139 / 89$ & 2.42 & 44,214 & $407,805.67$ & $1548(3.50)$ & $3.80(0.73-6.86)$ & 2.24 & 9983 & $54,240.97$ & $220(2.20)$ & $4.06(3.52-4.60)$ \\
\hline & & $\geq 140 / 90$ & 1.24 & 13,751 & $123,886.58$ & $1209(8.79)$ & $9.76(4.22-15.30)$ & 1.72 & 6949 & $38,376.21$ & $226(3.25)$ & $5.89(5.12-6.66)$ \\
\hline & $\mathrm{ACD}$ & $<130$ and $<80$ & 9.67 & 84,653 & $784,456.07$ & $254(0.30)$ & $0.32(0.00-2.54)$ & 8.19 & 20,903 & $11,2374.2$ & $60(0.29)$ & $0.53(0.40-0.67)$ \\
\hline & & $130 / 80-139 / 89$ & 8.42 & 44,214 & $407,805.67$ & $279(0.63)$ & $0.68(0.00-3.75)$ & 6.28 & 9983 & $54,240.97$ & $38(0.38)$ & $0.70(0.48-0.92)$ \\
\hline & & $\geq 140 / 90$ & 2.75 & 13,751 & $123,886.58$ & $286(2.08)$ & $2.31(0.00-7.87)$ & 3.5 & 6949 & $38,376.21$ & $57(0.82)$ & $1.49(1.10-1.87)$ \\
\hline & MACE & $<130$ and $<80$ & 9.68 & 84,653 & $784,456.07$ & $120(0.14)$ & $0.15(0.00-2.37)$ & 9.5 & 20,903 & $11,2374.2$ & $32(0.15)$ & $0.29(0.19-0.38)$ \\
\hline & & $130 / 80-139 / 89$ & 9.84 & 44,214 & $407,805.67$ & $136(0.31)$ & $0.33(0.00-3.40)$ & 9.5 & 9983 & $54,240.97$ & $19(0.19)$ & $0.35(0.19-0.51)$ \\
\hline & & $\geq 140 / 90$ & 4.08 & 13,751 & $123,886.58$ & $150(1.09)$ & $1.21(0.00-6.78)$ & 6.5 & 6949 & $38,376.21$ & $29(0.42)$ & $0.76(0.48-1.03)$ \\
\hline \multirow{9}{*}{$\begin{array}{c}2017 \\
\text { ACC/AHA }\end{array}$} & $\mathrm{ACM}$ & $<120$ and $<80$ & 3.51 & 53,374 & $494,529.82$ & 1051 (1.97) & $2.13(0.00-4.91)$ & 2.74 & 17,989 & $97,368.52$ & 259 (1.44) & $2.66(2.34-2.98)$ \\
\hline & & $120-129$ and $<80$ & 3.67 & 31,279 & $289,926.25$ & $803(2.57)$ & $2.77(0.00-6.41)$ & 1.72 & 2914 & $15,005.72$ & $93(3.19)$ & $6.20(4.94-7.45)$ \\
\hline & & $\geq 130 / 80$ & 1.83 & 57,965 & $531,692.25$ & $2757(4.76)$ & $5.19(2.50-7.87)$ & 1.92 & 16,932 & $92,617.18$ & $446(2.63)$ & $4.82(4.37-5.26)$ \\
\hline & $\mathrm{ACD}$ & $<120$ and $<80$ & 9.51 & 53,374 & $494,529.82$ & $123(0.23)$ & $0.25(0.00-3.04)$ & 8.21 & 17,989 & $97,368.52$ & $35(0.19)$ & $0.36(0.24-0.48)$ \\
\hline & & $120-129$ and $<80$ & 6.59 & 31,279 & $289,926.25$ & $131(0.42)$ & $0.45(0.00-4.09)$ & 3.28 & 2914 & $15,005.72$ & $25(0.86)$ & $1.67(1.01-2.32)$ \\
\hline & & $\geq 130 / 80$ & 6.17 & 57,965 & $531,692.25$ & $565(0.97)$ & $1.06(0.00-3.75)$ & 4.78 & 16,932 & $92,617.18$ & $95(0.56)$ & $1.03(0.82-1.23)$ \\
\hline & MACE & $<120$ and $<80$ & 8.76 & 53,374 & $494,529.82$ & $57(0.11)$ & $0.12(0.00-2.90)$ & 9.5 & 17,989 & $97,368.52$ & $18(0.10)$ & $0.19(0.10-0.27)$ \\
\hline & & $120-129$ and $<80$ & 9.68 & 31,279 & $289,926.25$ & $63(0.20)$ & $0.22(0.00-3.86)$ & 4.91 & 2914 & $15,005.72$ & $14(0.48)$ & $0.93(0.44-1.42)$ \\
\hline & & $\geq 130 / 80$ & 9.34 & 57,965 & $531,692.25$ & $286(0.49)$ & $0.54(0.00-3.23)$ & 8.16 & 16,932 & $92,617.18$ & $48(0.28)$ & $0.51(0.37-0.67)$ \\
\hline
\end{tabular}

Abbreviations: JNC7, the Joint National Committee 7; 2017 ACC/AHA, 2017 American College of Cardiology/American Heart Association; ACM: All-cause mortality; ACD: All cardiovascular

death; MACE: Major cardiovascular death; CI: Confidence interval. 
Table 3. Hazard ratios for all-cause mortality, all cardiovascular death, and major cardiovascular death according to JNC7 and 2017 ACC/AHA guidelines.

\begin{tabular}{|c|c|c|c|c|c|c|c|}
\hline \multicolumn{3}{|c|}{ Guidelines } & \multicolumn{5}{|c|}{ 2006-2015 NHIS-HEALS } \\
\hline & & Groups & $\begin{array}{c}\text { Adjusted HR } \\
(95 \% \mathrm{CI})^{1}\end{array}$ & $\begin{array}{l}p \text {-Value } \\
\text { for Trend }\end{array}$ & Pairwise & Compariso & -Value \\
\hline \multirow{9}{*}{ JNC7 } & $\mathrm{ACM}$ & $<130$ and $<80$ & 1 & \multirow{3}{*}{$<0.0001$} & ref & & \multirow[b]{3}{*}{ ref } \\
\hline & & $130 / 80-139 / 89$ & $1.10(1.02-1.18)$ & & 0.0102 & ref & \\
\hline & & $\geq 140 / 90$ & $1.84(1.70-2.00)$ & & $<0.0001$ & $<0.0001$ & \\
\hline & $\mathrm{ACD}$ & $<130$ and $<80$ & 1 & \multirow{3}{*}{$<0.0001$} & ref & & \multirow{3}{*}{ Ref } \\
\hline & & $130 / 80-139 / 89$ & $1.36(1.13-1.62)$ & & 0.0011 & ref & \\
\hline & & $\geq 140 / 90$ & $2.72(2.26-3.27)$ & & $<0.0001$ & $<0.0001$ & \\
\hline & MACE & $<130$ and $<80$ & 1 & & ref & & \\
\hline & & $130 / 80-139 / 89$ & $1.40(1.08-1.81)$ & $<0.0001$ & 0.012 & ref & \\
\hline & & $\geq 140 / 90$ & $3.25(2.51-4.21)$ & & $<0.0001$ & $<0.0001$ & Ref \\
\hline \multirow{9}{*}{$\begin{array}{c}2017 \\
\text { ACC/AHA }\end{array}$} & $\mathrm{ACM}$ & $<120$ and $<80$ & 1 & \multirow{3}{*}{$<0.0001$} & ref & & \multirow[b]{3}{*}{ Ref } \\
\hline & & $120-129$ and $<80$ & $0.77(0.69-0.84)$ & & $<0.0001$ & ref & \\
\hline & & $\geq 130 / 80$ & $1.15(1.06-1.24)$ & & 0.0005 & $<0.0001$ & \\
\hline & \multirow[t]{3}{*}{$\mathrm{ACD}$} & $<120$ and $<80$ & 1 & \multirow{3}{*}{$<0.0001$} & ref & & \\
\hline & & $120-129$ and $<80$ & $0.99(0.77-1.28)$ & & 0.9098 & ref & \\
\hline & & $\geq 130 / 80$ & $1.76(1.42-2.17)$ & & $<0.0001$ & $<0.0001$ & Ref \\
\hline & \multirow[t]{3}{*}{ MACE } & $<120$ and $<80$ & 1 & \multirow{3}{*}{$<0.0001$} & ref & & \\
\hline & & $120-129$ and $<80$ & $1.14(0.79-1.65)$ & & 0.4968 & ref & \\
\hline & & $\geq 130 / 80$ & $2.09(1.53-2.84)$ & & $<0.0001$ & $<0.0001$ & Ref \\
\hline \multicolumn{3}{|c|}{ Guidelines } & \multicolumn{5}{|c|}{ 2007-2015 KNAHES Cohorts } \\
\hline & & Groups & $\begin{array}{c}\text { Adjusted HR } \\
(95 \% \mathrm{CI})^{2}\end{array}$ & $\begin{array}{l}p \text { for } \\
\text { Trend }\end{array}$ & Pairwise & Compariso & -Value \\
\hline \multirow{9}{*}{ JNC7 } & $\mathrm{ACM}$ & $<130$ and $<80$ & 1 & \multirow{3}{*}{0.0234} & ref. & & \\
\hline & & $130 / 80-139 / 89$ & $0.97(0.82-1.15)$ & & 0.7352 & ref. & \\
\hline & & $\geq 140 / 90$ & $1.24(1.05-1.46)$ & & 0.0128 & 0.0095 & ref. \\
\hline & $\mathrm{ACD}$ & $<130$ and $<80$ & 1 & \multirow{3}{*}{0.0022} & ref. & & \\
\hline & & $130 / 80-139 / 89$ & $1.00(0.66-1.51)$ & & 0.9963 & ref. & \\
\hline & & $\geq 140 / 90$ & $1.83(1.26-2.65)$ & & 0.0015 & 0.0041 & ref. \\
\hline & \multirow[t]{3}{*}{ MACE } & $<130$ and $<80$ & 1 & \multirow{3}{*}{0.0482} & ref. & & \\
\hline & & $130 / 80-139 / 89$ & $0.91(0.51-1.62)$ & & 0.7520 & ref. & \\
\hline & & $\geq 140 / 90$ & $1.73(1.04-2.90)$ & & 0.0361 & 0.0298 & ref. \\
\hline \multirow{9}{*}{$\begin{array}{c}2017 \\
\text { ACC/AHA }\end{array}$} & $\mathrm{ACM}$ & $<120$ and $<80$ & 1 & \multirow{3}{*}{0.0592} & ref. & & \\
\hline & & $120-129$ and $<80$ & $1.37(1.08-1.74)$ & & 0.0101 & ref. & \\
\hline & & $\geq 130 / 80$ & $1.18(1.01-1.38)$ & & 0.0407 & 0.1913 & ref. \\
\hline & $\mathrm{ACD}$ & $<120$ and $<80$ & 1 & \multirow{3}{*}{0.0051} & ref. & & \\
\hline & & $120-129$ and $<80$ & $2.59(1.54-4.36)$ & & 0.0003 & ref. & \\
\hline & & $\geq 130 / 80$ & $1.86(1.25-2.77)$ & & 0.0022 & 0.1446 & ref. \\
\hline & MACE & $<120$ and $<80$ & 1 & \multirow{3}{*}{0.0654} & ref. & & \\
\hline & & $120-129$ and $<80$ & $2.88(1.42-5.87)$ & & 0.0034 & ref. & \\
\hline & & $\geq 130 / 80$ & $1.81(1.04-3.15)$ & & 0.0363 & 0.1271 & ref. \\
\hline
\end{tabular}

${ }^{1}$ Adjusted for age, sex, BMI, physical activity, household income, smoking status, alcohol status, fasting serum glucose, and total cholesterol. ${ }^{2}$ Adjusted for age, sex, BMI. ACM: All-cause mortality; ACD: All cardiovascular death; MACE: Major cardiovascular death; HR: Hazard ratio; CI: Confidence interval.

To evaluate the potential discriminatory ability of the two guidelines for clinical outcomes, Harrell's C-indexes were calculated and are presented in Table 4. These results suggest that the JNC7 guidelines have a greater discrimination ability in the NHIS-HEALS dataset and show no significant difference between the two guidelines in the KNAHES dataset. 
Table 4. Discrimination ability for all-cause mortality, all cardiovascular death, and major cardiovascular death according to JNC7 and 2017 ACC/AHA guidelines.

\begin{tabular}{ccccccc}
\hline \multicolumn{3}{c}{ 2006-2015 NHIS-HEALS } & \multicolumn{2}{c}{ 2007-2015 KNAHES Cohort } \\
\hline & JNC 7 & 2017 ACC/AHA & $p$-Value & JNC 7 & 2017 ACC/AHA & $p$-Value \\
\hline ACM & $0.622(0.613-0.63)$ & $0.605(0.597-0.612)$ & $<0.0001$ & $0.566(0.546-0.584)$ & $0.57(0.551-0.586)$ & 0.4237 \\
ACD & $0.682(0.666-0.704)$ & $0.656(0.643-0.673)$ & $<0.0001$ & $0.607(0.561-0.649)$ & $0.613(0.575-0.645)$ & 0.5854 \\
MACE & $0.692(0.667-0.716)$ & $0.663(0.644-0.686)$ & $<0.0001$ & $0.582(0.533-0.636)$ & $0.596(0.544-0.639)$ & 0.3861 \\
\hline
\end{tabular}

JNC7, the Joint National Committee 7; 2017 ACC/AHA, 2017 American College of Cardiology/American Heart Association; ACM: All-cause mortality; ACD: All cardiovascular death; MACE: Major cardiovascular death. $p$-values were calculated using Harrell's c-index.

\section{Discussion}

Hypertension is a major risk factor for cardiovascular morbidity and mortality [11]. Due to the association of hypertension and BP, optimal BP thresholds and treatment goals have long been a source of debate. Although the recent 2017 ACC/AHA guidelines recommend treating patients to reduce $\mathrm{SBP} / \mathrm{DBP}$ to $<130 / 80 \mathrm{~mm} \mathrm{Hg}$ [1], it is still uncertain whether such aggressive BP control results in improved clinical outcomes. Moreover, there is little direct evidence to guide the choice of target BP in Asian populations.

To address these unanswered questions, we compared clinical outcomes using the JNC7 and 2017 ACC/AHA guidelines in two nationally representative Korean population datasets. Based on the JNC7 guidelines, there was a linear association between BP levels and survival outcomes in both datasets. Similarly, the HTN group had unfavorable survival outcomes as reflected by warranty periods and event rates per 1000 person-years, regardless of dataset. In addition, adjusted HRs calculated using the JNC7 guidelines were able to differentiate the HTN group from the elevated and normal BP groups in all clinical outcomes. In contrast, the 2017 ACC/AHA guidelines showed inconsistent survival outcomes according to BP classification in the two datasets. Moreover, the JNC7 guidelines had more discrimination ability for all survival outcomes than the 2017 ACC/AHA guidelines according to Harrell's c-indexes in the NHIS-HEALS dataset.

It is uncertain why our results differ from the SPRINT study and recent meta-analyses [12] with emphasis on the strict BP lowering treatment. The differences may be explained in part by countryor ethnicity-specific factors and differing health environments. For example, the cutoff value for the definition of obesity is lower for Asian populations than for Western populations [13]. Asian populations tend to have a high salt intake, salt sensitivity, masked HTN, and nocturnal HTN [13]. Also, exaggerated morning BP surges are more frequent in Asian populations compared with Western populations [13]. Therefore, the influence of BP on health outcomes may differ among Asians and Westerners [14]. Future studies are warranted to clarify the possible mechanisms underlying the differences related to race and ethnicity.

Additionally, several studies in Western populations demonstrated inconsistencies with the SPRINT study. The SHEP (Systolic Hypertension in the Elderly Program) trial postulated that less intensive BP control may be more suitable for older patients, [15] and a pooled analysis of the ONTARGET and TRANSCEND trials suggested that excessive reduction of BP (SBP $<120 \mathrm{~mm} \mathrm{Hg}$ ) increased mortality and cardiovascular events compared to SBP between 120 and $140 \mathrm{~mm} \mathrm{Hg}$ [16]. Furthermore, the post hoc analysis of the SPRINT trial found that even those with a lower baseline CVD risk of $<18.2 \%$ experienced more harm than benefit following strict BP lowering treatment [17]. The strict BP thresholds of the new guidelines may be more appropriate for use in younger patients and in patients with higher CVD risk than those of the JNC7 guidelines. This is supported by recent epidemiologic data from the NHIS-HEALS cohort in young hypertensive patients as well as in patients with atrial fibrillation and HTN. Among young Korean adults with hypertension defined by the 2017 ACC/AHA guidelines, the risk of subsequent cardiovascular disease events was elevated compared to those with normal BP, [18] and BP between 120 and 129/<80 $\mathrm{mm} \mathrm{Hg}$ was found to be the optimal BP treatment target for patients with atrial fibrillation undergoing hypertension treatment [19]. Therefore, 
it is reasonable that the benefits of intensive BP reduction according to the new guidelines may differ according to age and associated cardiovascular risks. In our study, we used two independent nationwide cohort databases, which enables a high degree of generalization of our results. The JNC7 guidelines showed consistent results and a linear association between BP level and survival outcome; however, the 2017 ACC/AHA guidelines failed to find a linear relationship between all-cause or cardiovascular mortality and BP classifications in the two datasets.

This study has several limitations. First, although BP measurement equipment in all health examination institutions received quality assessment according to the Basic Act on National Health Examination, the lack of device uniformity and single visit measurements may have introduced some variability into our results. Unfortunately, it was impossible to measure home BP monitoring and ambulatory BP monitoring in routine evaluations. Second, because the current study was observational, confounding factors could result in over- or under-estimation of the effect of BP on clinical outcomes. Third, we did not account for antihypertensive medications that may affect the all-cause or cardiovascular mortality and could not conclude whether those who took antihypertensive medications following the JNC7 had a significantly lower mortality than those following the 2017 ACC/AHA guidelines. Also, we did not consider the mortality of heart failure that could create structural modification of the left ventricle [20]. Fourth, there is a possibility of sampling bias as our results were not weighted due to a lack of information on sampling weights in the data obtained from the NHIS-HEALS and KNHANES. Finally, our study population was composed of only Korean adults, so the results may not be generalizable to other races or ethnicities. Further investigations are required to evaluate and select the optimal BP guidelines and to determine the applicability of a new $\mathrm{BP}$ threshold with consideration given to the differences in healthcare environments.

Nevertheless, our results represent real word data and reflect practical clinical conditions. To our knowledge, this is the first study to directly compare the two guidelines in terms of all-cause and cardiovascular mortality.

\section{Conclusions}

Unlike the 2017 ACC/AHA guidelines, the JNC7 guidelines demonstrated a linear association between BP levels and survival outcomes in two nationwide datasets as well as good discrimination ability in all survival outcomes. Collectively, our results suggest that the JNC7 guidelines are more appropriate than the 2017 ACC/AHA guidelines in Korean populations.

Author Contributions: Conceptualization, H.-J.C. and J.-W.L.; Data curation, H.-S.L. and J.H.H.; Formal analysis, W.-J.C., H.-S.L., J.H.H., H.-J.C. and J.-W.L.; Funding acquisition, J.-W.L.; Investigation, W.-J.C., H.-S.L., H.-J.C. and J.-W.L.; Methodology, W.-J.C., H.-S.L., J.H.H., H.-J.C. and J.-W.L.; Validation, W.-J.C., H.-S.L. and J.H.H.; Writing-Original draft, W.-J.C. and H.-S.L.; Writing-Review \& editing, H.-J.C. and J.-W.L.

Funding: This work was supported by the Bio and Medical Technology Development Program through the National Research Foundation of Korea funded by the Ministry of Science, ICT, and Future Planning (NRF-2018R1D1A1B07049223), the Technology Innovation Program (20002781, A Platform for Prediction and Management of Health Risk Based on Personal Big Data and Lifelogging) funded by the Ministry of Trade, Industry \& Energy (MOTIE, Korea).

Acknowledgments: This work was supported by the pilot study of the Korea National Health and Nutrition Examination Survey linked Cause of death data by the Korea Centers for Disease Control and Prevention.

Conflicts of Interest: Authors declare no conflict of interest.

\section{References}

1. Whelton, P.K.; Carey, R.M.; Aronow, W.S.; Casey, D.E., Jr.; Collins, K.J.; Dennison Himmelfarb, C.; DePalma, S.M.; Gidding, S.; Jamerson, K.A.; Jones, D.W.; et al. 2017 ACC/AHA/AAPA/ABC/ACPM/AGS/APhA/ASH/ASPC/NMA/PCNA Guideline for the Prevention, Detection, Evaluation and Management of High Blood Pressure in Adults: Executive Summary: A Report of the American College of Cardiology/American Heart Association Task Force on Clinical Practice Guidelines. Circulation 2018, 138, 426-483. [CrossRef] 
2. Wright, J.T., Jr.; Williamson, J.D.; Whelton, P.K.; Snyder, J.K.; Sink, K.M.; Rocco, M.V.; Reboussin, D.M.; Rahman, M.; Oparil, S.; Lewis, C.E.; et al. A Randomized Trial of Intensive versus Standard Blood-Pressure Control. N. Engl. J. Med. 2015, 373, 2103-2116. [CrossRef] [PubMed]

3. Reboussin, D.M.; Allen, N.B.; Griswold, M.E.; Guallar, E.; Hong, Y.; Lackland, D.T.; Miller, E.P.R., 3rd; Polonsky, T.; Thompson-Paul, A.M.; Vupputuri, S. Systematic Review for the 2017 ACC/AHA/AAPA/ABC/ACPM/AGS/APhA/ASH/ASPC/NMA/PCNA Guideline for the Prevention, Detection, Evaluation, and Management of High Blood Pressure in Adults: A Report of the American College of Cardiology/American Heart Association Task Force on Clinical Practice Guidelines. Circulation 2018, 138, 595-616. [CrossRef]

4. Vaduganathan, M.; Pareek, M.; Qamar, A.; Pandey, A.; Olsen, M.H.; Bhatt, D.L. Baseline Blood Pressure, the 2017 ACC/AHA High Blood Pressure Guidelines, and Long-Term Cardiovascular Risk in SPRINT. JAMA Cardiol. 2018, 131, 956-960. [CrossRef] [PubMed]

5. Bundy, J.D.; Mills, K.T.; Chen, J.; Li, C.; Greenland, P.; He, J. Estimating the Association of the 2017 and 2014 Hypertension Guidelines With Cardiovascular Events and Deaths in US Adults: An Analysis of National Data. JAMA Cardiol. 2018, 3, 572-581. [CrossRef] [PubMed]

6. Ogihara, T.; Saruta, T.; Matsuoka, H.; Shimamoto, K.; Fujita, T.; Shimada, K.; Imai, Y.; Nishigaki, M. Valsartan in elderly isolated systolic hypertension (VALISH) study: Rationale and design. Hypertens. Res. 2004, 27, 657-661. [CrossRef] [PubMed]

7. Margolis, K.L.; O'Connor, P.J.; Morgan, T.M.; Buse, J.B.; Cohen, R.M.; Cushman, W.C.; Cutler, J.A.; Evans, G.W.; Gerstein, H.C.; Grimm, R.H., Jr.; et al. Outcomes of combined cardiovascular risk factor management strategies in type 2 diabetes: The ACCORD randomized trial. Diabetes Care 2014, 37, 1721-1728. [CrossRef] [PubMed]

8. Williams, B.; Mancia, G.; Spiering, W.; Agabiti Rosei, E.; Azizi, M.; Burnier, M.; Clement, D.L.; Coca, A.; de Simone, G.; Dominiczak, A.; et al. 2018 ESC/ESH Guidelines for the management of arterial hypertension: The Task Force for the management of arterial hypertension of the European Society of Cardiology and the European Society of Hypertension: The Task Force for the management of arterial hypertension of the European Society of Cardiology and the European Society of Hypertension. J. Hypertens. 2018, 36, 1953-2041. [CrossRef] [PubMed]

9. Kario, K.; Chen, C.-H.; Park, S.; Park, C.-G.; Hoshide, S.; Cheng, H.-M.; Huang, Q.-F.; Wang, J.-G. Consensus document on improving hypertension management in Asian patients, taking into account Asian characteristics. Hypertension 2018, 71, 375-382. [CrossRef] [PubMed]

10. Kweon, S.; Kim, Y.; Jang, M.-J.; Kim, Y.; Kim, K.; Choi, S.; Chun, C.; Khang, Y.-H.; Oh, K. Data resource profile: The Korea national health and nutrition examination survey (KNHANES). Int. J. Epidemiol. 2014, 43, 69-77. [CrossRef] [PubMed]

11. Nwankwo, T.; Yoon, S.S.; Burt, V.; Gu, Q. Hypertension among adults in the United States: National Health and Nutrition Examination Survey, 2011-2012. NCHS Data Brief. 2013, 133, 1-8.

12. Weiss, J.; Freeman, M.; Low, A.; Fu, R.; Kerfoot, A.; Paynter, R.; Motu'apuaka, M.; Kondo, K.; Kansagara, D. Benefits and harms of intensive blood pressure treatment in adults aged 60 years or older: A systematic review and meta-analysis. Ann. Intern. Med. 2017, 166, 419-429. [CrossRef] [PubMed]

13. Ihm, S.-H.; Bakris, G.; Sakuma, I.; Sohn, I.S.; Koh, K.K. Controversies in the 2017 ACC/AHA Hypertension Guidelines: Who Can Be Eligible for Treatments Under the New Guidelines?-An Asian Perspective. Circ. J. 2019, 83, 504-510. [CrossRef] [PubMed]

14. Collaboration, A.P.C.S. Blood pressure and cardiovascular disease in the Asia Pacific region. J. Hypertens. 2003, 21, 707-716.

15. Perry, H.M., Jr.; Davis, B.R.; Price, T.R.; Applegate, W.B.; Fields, W.S.; Guralnik, J.M.; Kuller, L.; Pressel, S.; Stamler, J.; Probstfield, J.L. Effect of treating isolated systolic hypertension on the risk of developing various types and subtypes of stroke: The Systolic Hypertension in the Elderly Program (SHEP). JAMA 2000, 284, 465-471. [CrossRef] [PubMed]

16. Bohm, M.; Schumacher, H.; Teo, K.K.; Lonn, E.M.; Mahfoud, F.; Mann, J.F.E.; Mancia, G.; Redon, J.; Schmieder, R.E.; Sliwa, K.; et al. Achieved blood pressure and cardiovascular outcomes in high-risk patients: Results from ONTARGET and TRANSCEND trials. Lancet 2017, 389, 2226-2237. [CrossRef] 
17. Phillips, R.A.; Xu, J.; Peterson, L.E.; Arnold, R.M.; Diamond, J.A.; Schussheim, A.E. Impact of Cardiovascular Risk on the Relative Benefit and Harm of Intensive Treatment of Hypertension. J. Am. Coll. Cardiol. 2018, 71, 1601-1610. [CrossRef] [PubMed]

18. Son, J.S.; Choi, S.; Kim, K.; Kim, S.M.; Choi, D.; Lee, G.; Jeong, S.-M.; Park, S.Y.; Kim, Y.-Y.; Yun, J.-M.; et al. Association of Blood Pressure Classification in Korean Young Adults According to the 2017 American College of Cardiology/American Heart Association Guidelines With Subsequent Cardiovascular Disease EventsCardiovascular Disease Rates Among Young Korean Adults by 2017 ACC/AHA Hypertension Guideline DefinitionsCardiovascular Disease Rates Among Young Korean Adults by 2017 ACC/AHA Hypertension Guideline Definitions. JAMA 2018, 320, 1783-1792. [CrossRef] [PubMed]

19. Kim, D.; Yang, P.S.; Kim, T.H.; Jang, E.; Shin, H.; Kim, H.Y.; Yu, H.T.; Uhm, J.S.; Kim, J.Y.; Pak, H.N.; et al. Ideal Blood Pressure in Patients With Atrial Fibrillation. J. Am. Coll. Cardiol. 2018, 72, 1233-1245. [CrossRef] [PubMed]

20. Severino, P.; Maestrini, V.; Mariani, M.V.; Birtolo, L.I.; Scarpati, R.; Mancone, M.; Fedele, F. Structural and myocardial dysfunction in heart failure beyond ejection fraction. Heart Fail. Rev. 2019, 1-9. [CrossRef]

(C) 2019 by the authors. Licensee MDPI, Basel, Switzerland. This article is an open access article distributed under the terms and conditions of the Creative Commons Attribution (CC BY) license (http://creativecommons.org/licenses/by/4.0/). 